\title{
DE LA RUPTURE FAMILIALE ET SOCIALE À LA RECONSTRUCTION D'UNE APPARTENANCE RELIGIEUSE LES NOUVELLES CATHOLIQUES DE ROUEN, DE 1685 À 1787
}

L'apogée des abjurations des protestants entre 1681 et 1686 a parfois tendance à occulter qu'elles se poursuivirent en réalité tout au long du XVIII $^{\mathrm{e}}$ siècle. En effet, la révocation de l'édit de Nantes, qui paracheva de longues persécutions juridiques et militaires ${ }^{1}$, avait instauré l'obligation de faire baptiser tous les enfants et de les faire instruire dans la religion catholique. L'implantation huguenote était forte dans les terres normandes jusqu'à la révocation (5,3\% des réformés du royaume), et elle le demeura bien après, en dépit de l'émigration vers les pays du Refuge ${ }^{2}$. La Normandie abritait encore vingt à vingt-cinq mille protestants à la fin du xviI ${ }^{\mathrm{e}}$ siècle, contre quarante mille au début du règne personnel de Louis XIV. En 1698, un rapport de l'intendant de la généralité de Rouen mentionnait mille six cent quarante-sept protestants, qualifiés de «nouveaux convertis", dans la seule capitale normande ${ }^{3}$, alors que, avant la révocation, on y recensait plus de quatre mille protestants ${ }^{4}$. La maison des Nouvelles Catholiques de Rouen, située dans la rue Étoupée de la paroisse Sainte-Croix-des-Pelletiers, convertit et forma de $1667{ }^{5}$ à 1792 un grand nombre de jeunes protestantes de toute la

1. Voir Didier Borsson et Hugues Daussy, Les protestants dans la France moderne, Paris, 2006 (Belin sup. Histoire), et Marianne Carbonnier-Burkard et Patrick Cabanel, Une histoire des protestants en France ( XVI ${ }^{e}-X X^{e}$ siècle), Paris, 1998.

2. Voir Luc Daireaux, "Réduire les huguenots 》 : protestants et pouvoirs en Normandie au XVII ${ }^{e}$ siècle, Paris, 2010 (Vie des huguenots, 57), et ID., « La Normandie protestante dans la seconde moitié du XviI ${ }^{\mathrm{e}}$ siècle ", dans Mickaël Augeron, Didier Poton et Bertrand Van RuymBeke (dir.), Les huguenots et l'Atlantique, vol. I, Pour Dieu, la cause ou les affaires, Paris, 2009 (Histoire maritime), p. 386-387.

3. Michel Join-Lambert, "La pratique religieuse dans le diocèse de Rouen sous Louis XIV ", dans Annales de Normandie, t. 3 (1953), p. 256.

4. L. Daireaux, "Postface. Protestants et protestantisme en Normandie du $\mathrm{xvI}^{\mathrm{e}}$ au $\mathrm{xVIII}^{\mathrm{e}}$ siècle : bilan historiographique ", dans Émile-G. LÉONARD, La résistance protestante en Normandie au XVIII siècle, Caen, 2005 (Cahiers des Annales de Normandie, 34), p. 113.

5. Arch. dép. de la Seine-Maritime, G 1289, titres de fondation des Nouvelles Catholiques de Rouen et déclaration de revenus, 1672-1723, et D 489 (registre des délibérations des Nouvelles Catholiques de Rouen, 1675-1698). Cette œuvre fut fondée à Rouen en 1667 par Robert Le Cornier, conseiller du roi et maître des comptes de Normandie, avec le soutien du curé de la 
Normandie, d'Angleterre, mais aussi quelques-unes de Montpellier, de Hollande, de Suisse, de Rhénanie-Palatinat, de Basse-Saxe, de Suède, et même de Nouvelle-Angleterre ${ }^{6}$.

Les débats historiques sur le caractère forcé ou volontaire des conversions comme ceux sur les vocations au XviI ${ }^{\mathrm{e}}$ siècle $^{7}$ demeurent vifs et paraissent insolubles. L'histoire des idées a pourtant permis de nuancer cette dialectique, en mettant en évidence le sens profondément différent de la notion de liberté avant la diffusion de l'esprit des Lumières. Dans l'historiographie du protestantisme, les études sur les abjurations forcées sont nombreuses et précises, mais la réflexion sur la conversion comme phénomène social a vu le jour récemment. La recherche sur ce thème est en plein développement dans plusieurs disciplines, dont les concepts s'enrichissent mutuellement (en particulier en histoire, philosophie, littérature, sociologie des religions, psychologie sociale) ${ }^{8}$. Dans cet article, la conversion est entendue comme le passage

paroisse Sainte-Croix-des-Pelletiers, Henry Cavelier. Jean Le Cornier, conseiller du roi et commissaire aux Requêtes du palais, ainsi que François de Bailleul, conseiller au parlement, signèrent également des contrats de fondation pour doter la communauté naissante. Celle-ci reçut l'autorisation des échevins le 17 février 1672, l'approbation de l'archevêque François Rouxel de Médavy le 9 juillet 1673 et les lettres patentes du roi en avril 1674, enregistrées au parlement en mai 1675.

6. Arch. dép. de la Seine-Maritime, D 487 (registre d'abjurations des Nouvelles Catholiques de Rouen, 1718-1783) et D 488 (registre d'abjurations des Nouvelles Catholiques de Rouen, 1783-1791).

7. Voir, entre autres, Barbara Diefendorf, «Give us Back our Children : Patriarchal Authority and Parental Consent to Religious Vocations in Early Counter-Reformation France ", dans Journal of Modern History, t. 68 (1996), p. 265-307 ; Dominique Dinet, Vocation et fidélité : le recrutement des réguliers dans les diocèses d'Auxerre, Langres et Dijon (XVII $\left.-X V I I I^{e}\right)$, Paris, 1988 (Coll. Histoire) ; Anne Jacobson SchutTe, By Force and Fear : Taking and Breaking Vows in Early Modern Europe, Ithaca (N. Y.)-Londres, 2011 ; Caroline LE MaO, "Entre vocation et devoir social : l'appel de Dieu dans le milieu parlementaire bordelais au temps de Louis XIV ", dans Denis Lopez, Charles Mazouer et Éric SuIre (éd.), La religion des élites au XVII siècle : actes du colloque du Centre de recherches sur le XVII siècle (1600-1700), en partenariat avec le Centre aquitain d'histoire moderne et contemporaine (université Michel-de-Montaigne Bordeaux-3, 30 novembre-2 décembre 2006), Tübingen, 2008 (Biblio 17, 175), p. 289-301 ; Alexandra Roger, «Contester l'autorité parentale: les vocations religieuses forcées au XVIII ${ }^{\mathrm{e}}$ siècle en France", dans Annales de démographie historique, no 125 (janv.-juin 2013), p. 43-67, et La vocation religieuse et sacerdotale en France (XVII-XIX siècles), Angers, 1979.

8. Le thème de la conversion a fait l'objet de plusieurs numéros des Cahiers d'études du religieux : $\mathrm{n}^{\circ} 8$ (2010) ["Expériences de conversion 》], nº spécial (2011) ["Actes de la journée Jeunes chercheurs sur la conversion »], $\mathrm{n}^{\circ} 9$ (2011) [ La conversion : Antiquité et Moyen Âge »], $n^{\circ} 10$ (2012) [ La conversion sur le temps long 》], et $\mathrm{n}^{\circ}$ spécial (2014) [ Les conversion religieuses en Méditerranée »]. Voir également Béatrice BakHouche, Isabelle FAvre et Vincente Fortier (éd.), Dynamiques de conversion, modèles et résistances : approches interdisciplinaires, Turnhout, 2012 (Bibl. de l'École des hautes études. Sciences religieuses, 155), 2012 ; D. Borsson et Élisabeth Pinto-Mathieu (dir.), La conversion : textes et réalités, Rennes, 2014 (Histoire) ; Pierre-Yves Brandt et Claude-Alexandre Fournier (éd.), La conversion religieuse : analyses psychologiques, anthropologiques et sociologiques, Genève, 2009 (Psychologie et spiritualité) ; Catherine Chalier, Le désir de conversion, Paris, 2011 ; Danièle Hervieu-LÉGer, La religion en mouvement : le pèlerin et le converti, [Paris], 1999 (Essais) ; Thomas LiEnHard et Isabelle Poutrin (éd.), Pouvoir politique et conversion religieuse, t. I, Normes et mots, Rome, 2017 (Coll. de l'École française de Rome, 542), en ligne à l'adresse : http://books.openedition.org/efr/3400 [site consulté le 3 mars 2018] ; Philippe MARTin et É. SuIRE (éd.), Les convertis : parcours religieux, parcours politiques, t. I, Période moderne, Paris, 2016 (Constitution de la modernité, 2), et Maria-Cristiana Pitassi et Daniela Solfaroli Camilloca (éd.), Les modes de la conversion confessionnelle à l'époque moderne: autobiographie, altérité et construction des identités religieuses, Florence, 2010 (Biblioteca della Rivista di storia e letteratura religiosa. Studi, 23). 
d'une religion à une autre, et non comme l'approfondissement de la foi au sein d'une religion reçue dès l'enfance. Les motifs de conversion des protestants au catholicisme, c'est-à-dire d'un groupe minoritaire à un groupe majoritaire, doivent être appréhendés différemment des conversions vers le protestantisme, car le fait de se fondre dans la masse en se soumettant à une religion officielle permettait de se mettre en sécurité, tandis que l'insécurité guettait ceux qui s'affiliaient à une confession minoritaire et persécutée. L'histoire du xviII $^{\mathrm{e}}$ siècle permet de s'interroger sur les conversions contraintes, résignées, difficilement acceptées, ou bien instrumentalisées. Penser le phénomène de la conversion avec des concepts issus des sciences sociales permet aux historiens de parvenir à une compréhension plus précise et plus complexe des intentions des acteurs qui choisirent de changer d'appartenance religieuse. Il paraît donc pertinent d'analyser les facteurs sociologiques qui ont amené ces personnes à se convertir.

La maison des Nouvelles Catholiques de Rouen constitue un observatoire intéressant pour envisager une nouvelle approche de la conversion au XVIII ${ }^{\mathrm{e}}$ siècle, grâce à un corpus de sources variées. Les archives de la communauté comportent deux registres d'abjurations offrant une série continue de 1718 à $1791^{9}$, que l'on peut confronter aux états trimestriels envoyés entre 1720 et 1725 (série continue) et entre 1750 et 1778 (série discontinue) par la supérieure des Nouvelles Catholiques de Rouen pour recevoir les pensions prises sur le Trésor royal ${ }^{10}$. Ces deux types de sources ne se recoupent que partiellement ${ }^{11}$, mais peuvent être exploitées indépendamment. La première renseigne, pour chaque nouveau converti, la date d'abjuration et l'identité (nom, prénom, âge, père et mère, paroisse d'origine). Les registres font état de trois cent vingt et une abjurations, dont trois cents de femmes, presque toutes ayant eu lieu dans l'église des Nouvelles Catholiques. Tous étaient protestants, sauf Esther Pimentel, qui était juive ${ }^{12}$. Les états dressés par la supérieure de la maison des Nouvelles Catholiques de Rouen, quant à eux, permettent d'identifier cent trente pensionnaires avec leurs nom et prénom, leur âge et, seulement pour la série de 1750 à 1778, la date de leur entrée dans la maison. On peut ainsi estimer l'âge à l'entrée et à la sortie de l'institut et, pour une part de l'échantillon, la durée du placement. Quant aux Constitutions de la communauté, elles éclairent les modalités de la réception des

9. Arch. dép. de la Seine-Maritime, D 487 et 488.

10. Arch. nat., TT 264, dossier 25 («Estat de ce qui est deü à la maison des Nouvelles Catholiques de Rouen [...] pour la pension des personnes qui demeurent dans ladite maison qui se font instruire à la foy et professent la religion catholique, apostolique et romaine qui y ont esté envoyées par des ordres de roy et de messieurs les intendants », 1720-1725), et TT 296, dossier 5 ( Estat des filles qui ont esté mises par ordre du roy dans la maison des Nouvelles Catholiques de Rouen pour y estres instruites des vérités de la religion et y abjurer leurs erreurs et dont Sa Majesté paye les pensions sur le Trésor royal pendant le temps de leur instruction », 1750-1756, 1759-1764, 1769-1770 et 1776-1778).

11. $20 \%$ environ des pensionnaires du roi entre 1720 à 1725 figurent dans la base des abjurations, et $80 \%$ de celles qui ont été dans la maison des Nouvelles Catholiques entre 1750 et 1778.

12. Arch. dép. de la Seine-Maritime, D 487, copie de l'acte de baptême d'Esther Pimentel, renommée Marie-Dominique-Adélaïde, 18 avril 1772. 
pensionnaires ${ }^{13}$. Parallèlement, la correspondance de l'intendant de la généralité de Rouen met en évidence son rôle dans leur placement ${ }^{14}$. Par conséquent, plusieurs trajectoires de nouvelles catholiques se dessinent, offrant la possibilité d'analyser les facteurs sociologiques de leur passage d'un groupe confessionnel à un autre, et les conséquences sur leur intégration sociale, médiatisée par la reconstruction de leur appartenance religieuse. À ce titre, une étude ciblée sur les filles et les femmes permet de se demander si les facteurs de leur conversion ont pu parfois revêtir des spécificités genrées. Les conversions au catholicisme des jeunes filles protestantes entre 1685 et 1787 peuvent-elles s'expliquer par un processus de marginalisation sociale aboutissant à la nécessité de se réinsérer?

La marginalisation sociale résultant d'un enlèvement ou d'un abandon a constitué une cause de la conversion religieuse des filles aux xviI et XVIII $^{\mathrm{e}}$ siècles. Les catholiques chargés par les autorités politiques et religieuses de la conversion de la jeunesse dans le royaume de France ont usé d'une stratégie d'isolement pour obtenir la conversion des enfants, en particulier celle des filles. Finalement, la conversion au catholicisme était synonyme d'une redéfinition de l'identité par la recomposition des liens sociaux qu'elle provoquait. Elle peut donc être envisagée comme outil de réinsertion sociale.

\section{La rupture familiale : des familles divisées par les enlèvements et les abjurations contraintes}

Les premiers enlèvements d'enfants protestants débutèrent dès la fin du $\mathrm{xvi}^{\mathrm{e}}$ siècle, à la suite de l'édit de Nemours de 1585, pour se prolonger jusqu'à la veille de la Révolution française. Dans le cadre de la politique d'unité religieuse de Louis XIV, une déclaration royale du 17 juin 1681 autorisa la conversion des plus jeunes dès l'âge de 7 ans, là où la législation les protégeait auparavant jusqu'à 14 ans pour les garçons et 12 ans pour les filles. Cet acte, qui légitimait les enlèvements d'enfants organisés par les intendants avec l'appui des officiers locaux et des archevêques, fit grossir les effectifs dans les établissements de propagation de la foi. L'arrêt du 16 décembre 1685 imposa par la suite aux parents protestants et «nouveaux convertis » d'élever leurs enfants dans la religion catholique. En cas de non-respect de la loi, les enfants âgés de 5 à 16 ans étaient enlevés à leur famille. Les juifs étaient également concernés par cet arrêt, mais, le 15 juillet 1728, une ordonnance signée à Versailles fit « défense aux maisons religieuses de recevoir les enfants des juifs sous prétexte de religion avant l'âge de douze ans ", leur accordant ainsi une protection spéciale en élevant l'âge en dessous duquel l'abjuration n'était pas

13. Constitutions pour la maison des Nouvelles Catholiques de Rouen, Rouen, PhilippePierre Cabut, 1731. Un exemplaire de cet ouvrage est conservé aux arch. dép. de la SeineMaritime, sous la cote D 482.

14. Arch. dép. de la Seine-Maritime, C 978 (correspondance relative aux ordres du roi pour faire recevoir aux Nouvelles Catholiques de Rouen des jeunes filles de la religion prétendue réformée, 1700-1787). 
considérée comme valable. Ces enfants étaient placés dans des instituts de propagation de la foi tels que les Nouveaux et Nouvelles Catholiques, les Filles de la Foi, l'Union chrétienne ${ }^{15}$, mais aussi dans divers couvents d'ursulines, de bénédictines, de visitandines pour les filles, chez les jésuites ou les doctrinaires pour les garçons ${ }^{16}$, voire dans des maisons de force ${ }^{17}$. À Rouen, les protestantes étaient placées prioritairement dans la maison des Nouvelles Catholiques, mais d'autres se répartissaient dans différents monastères. À la fin du Xvie siècle, Marie Le Boulanger et Marie Bunel se trouvaient chez les dominicaines du Précieux-Sang, dans le faubourg Cauchoise ${ }^{18}$. Plusieurs étrangères étaient pensionnaires chez les visitandines : Marie Borchers, luthérienne et fille de Jacob Borchers, négociant originaire de Hambourg devenu consul de Suède à Rouen, abjura le 23 février 1761 ; Sarah O’Rork, une anglicane née dans le comté de Suffolk, se convertit le 18 décembre 1769 ; Elizabeth Hawcourt et Arabélie Linfield, venues d'Angleterre, abjurèrent toutes les deux l'anglicanisme le 4 juin $1772^{19}$. Ce même jour, une autre cérémonie se déroulait dans l'église des clarisses anglaises ${ }^{20}$ pour l'abjuration d'Esther Bent, presbytérienne. La raison d'être de ce monastère de religieuses anglaises était la perpétuation du catholicisme dans l'attente d'un retour à la tolérance en Angleterre, voire de sa réintégration dans l'Église romaine ${ }^{21}$. C' est pourquoi elles étaient les plus aptes à convertir au catholicisme leurs compatriotes, telles Marie Wild, Marthe Gayle, Marie Lancaster et Sarah Hores, qui abjurèrent respectivement

15. Ces établissements religieux dédiés à la conversion et à l'instruction des protestants avaient été fondés au cours du XviI ${ }^{\mathrm{e}}$ siècle. Ces couvents de filles séculières ne doivent pas être confondus avec les compagnies de la Propagation de la foi - dont le titre officiel était compagnies de l'Exaltation de la Sainte-Croix —, gérées par des ecclésiastiques séculiers et des laïcs. La première compagnie de la Propagation de la foi fut fondée à Paris par le père capucin Hyacinthe le 14 septembre 1632. Un certain nombre de dames dévotes collaboraient à cette compagnie et dirigèrent la maison des Nouvelles Catholiques de Paris, créée en 1634, jusqu'à ce que celle-ci adopte une organisation autonome sous la forme d'un couvent de filles séculières dans les années 1670. Voir Catherine Martin, Les compagnies de la Propagation de la foi (1632-1685, Paris, Grenoble, Aix, Lyon, Montpellier) : étude d'un réseau d'associations fondé en France au temps de Louis XIII pour lutter contre l'hérésie, des origines à la révocation de l'édit de Nantes, Genève, 2000 (Travaux du Grand Siècle, 16), p. 9, 35-36, 43 et 125-131.

16. On trouve une liste des «institutions et des maisons pour recevoir les enfants enlevés à leurs parents protestants " dans Alain Joblin, Dieu, le juge et l'enfant : l'enlèvement des enfants protestants en France (XVII ${ }^{e}$ XVIII ${ }^{e}$ siècles), Arras, 2010 (Histoire), p. 168-169.

17. C'est le cas, par exemple, du couvent de la Magdeleine à Bordeaux. Voir Aude Loriaud, "De la conversion à la prise de voile $\mathrm{au}_{\text {XVIII }}^{\mathrm{e}}$ siècle : parcours de sept juives et protestantes au sein de communautés catholiques bordelaises ", dans Nicolas Champ et É. Suire (éd.), Les appartenances religieuses : confessions, sensibilités et particularismes dans l'histoire du Sud-Ouest (actes du LXIV congrès de la Fédération historique du Sud-Ouest, Saintes, 2011), Bordeaux, 2012, p. 141-152.

18. Arch. nat., TT 264, dossier 16, lettre de la supérieure du monastère Saint-Dominique du faubourg Cauchoise demandant une pension pour la prise d'habit de Marie Le Boulanger, nouvelle convertie, 24 janvier 1688, et placet adressé par Marie Le Boulanger et Marie Bunel au marquis de Châteauneuf, 24 mars 1688.

19. Arch. dép. de la Seine-Maritime, D 487. Les cérémonies des trois premières converties citées se déroulèrent dans l'église du premier monastère de la Visitation et dans le second monastère de la Visitation pour la dernière.

20. Appelées "Gravelines ", du nom de la ville de Flandre où elles étaient implantées avant de fuir la guerre en 1644 .

21. Voir Claire WALKer, Gender and Politics in Early Modern Europe : English Convents in France and the Low Countries, Basingstoke-New York, 2003 (Early Modern History). 
les $1^{\text {er }}$ juin 1759, 25 mars $1761,1^{\text {er }}$ décembre 1763 et 17 juin $1770{ }^{22}$. Il est fort possible également que les ursulines et la Congrégation de Notre-Dame, qui se vouaient à l'enseignement, instruisirent dans la foi catholique des filles et des femmes protestantes à Rouen.

D'après Alain Joblin, deux fois plus de filles que de garçons furent victimes d'enlèvements dans le royaume de France ${ }^{23}$. Elles furent beaucoup plus ciblées, car la Réforme catholique promue par le concile de Trente plaçait ses espoirs dans l'enseignement comme moyen de contrer l'essor du protestantisme et d'ancrer plus profondément le catholicisme dans les familles. Dans le cadre de ce projet, l'éducation des futures mères de famille prit une place importante. En effet, au xvi ${ }^{\mathrm{e}}$ siècle, le clergé comprit — à la suite de Luther 24 et des protestants, qui développèrent un réseau important d'écoles - que si l'on élevait une fille dans la religion catholique, toute une famille en bénéficierait, puisque cette future mère éduquerait ses propres enfants. La supériorité du nombre d'enlèvements de filles s'expliquerait donc par cette idéologie d'un renouvellement de la société par les femmes. Plus généralement, cette forme de « captation » des enfants afin de les affilier à l'Église catholique reposait sur des normes canoniques et un corpus juridique et théologique légitimant les conversions forcées des enfants de parents juifs, réformés ou musulmans, ou dont un des parents au moins était chrétien ${ }^{25}$. Malgré tout, le droit du roi à faire enlever des enfants pour leur donner une éducation religieuse faisait controverse ${ }^{26}$.

Les enfants de familles non catholiques étaient mis à l'écart dans des couvents pour les éloigner de leur milieu familial et les amener à abandonner leur identité religieuse première afin de se fondre ensuite dans la société que l'État voulait exclusivement catholique. Les communautés religieuses qui avaient pour mission de convertir les non-catholiques étaient donc des creusets d'une redéfinition de l'appartenance religieuse. Cet enfermement, bien que temporaire, semble avoir été une stratégie employée plus souvent à l'égard des filles que des garçons. En effet, ceux-ci subirent également des placements contraints dans des communautés religieuses, en particulier chez les jésuites et les doctrinaires, mais la rareté des maisons spécialisées dans la conversion des garçons par rapport à l'abondance de celles qui étaient destinées aux filles est flagrante (dans un rapport de deux pour dix environ ${ }^{27}$ ). Comme le seul fait d'abjurer n'était pas considéré comme suffisant, les nouvelles converties

22. Arch. dép. de la Seine-Maritime, D 487.

23. A.Joblin, Dieu, le juge et l'enfant..., p. 39 et 44. Cette estimation repose sur un échantillon de mille deux cents cas d'enlèvements retenus pour une période de soixante-quatre années, échelonnées de 1665 à 1783, survenus dans l'Ouest et le Sud du royaume, outre la ville de Paris.

24. Dès 1524, Luther insistait sur la nécessité d'instruire autant les filles que les garçons. Voir P. CABANEL et André ENCREvÉ, " De Luther à la loi Debré : protestantisme, école et laïcité ", dans Histoire de l'éducation, t. 110 (mai 2006), p. 5-21, et D. Dinet, "L'éducation des filles, de la fin du $18^{\mathrm{e}}$ siècle jusqu'en 1918 ", dans Revue des sciences religieuses, t. 85 (2011), p. 457-490.

25. Voir I. PouTRIN, « La captation de l'enfant de converti : l'évolution des normes canoniques à la lumière de l'antijudaïsme des $\mathrm{XvI}^{\mathrm{e}}$-xviII ${ }^{\mathrm{e}}$ siècles ", dans Revue d'histoire moderne et contemporaine, t. $62, \mathrm{n}^{\circ} 2-3$ (avr.-sept. 2015), p. 40-62.

26. A. Joblin, Dieu, le juge et l'enfant..., p. 61-74.

27. Proportion calculée à partir du nombre de maisons de Nouvelles et Nouveaux Convertis, et celles des Nouvelles et Nouveaux Catholiques figurant dans la liste des «institutions et des maisons pour recevoir les enfants enlevés à leurs parents protestants » (ibid., p. 168-169). 
pouvaient rester jusqu'à ce que la supérieure de la communauté jugeât que leur foi catholique était assez ferme pour leur redonner leur liberté. La durée de leur séjour chez les Nouvelles Catholiques entre 1750 et 1778 était en moyenne de quatre ans ${ }^{28}$, alors que les Constitutions des Nouvelles Catholiques prévoyaient en théorie une période d'instruction de trois mois seulement ${ }^{29}$. La durée de leur placement — ou de leur enfermement, comme on préférera - pouvait aller au-delà de dix années ${ }^{30}$, voire plus de seize années dans les cas de Marie-Luce-Benoîte Gaillard, enfermée à l'âge de 9 ans, et Marie-Esther Doyerre, enfermée à 6 ans, qui étaient encore dans la maison des Nouvelles Catholiques de Rouen en 1778, alors âgées respectivement de 23 et 22 ans. L'absence d'états postérieurs à 1778 empêche de savoir combien de temps elles durent encore attendre avant de retrouver leur liberté. Les registres d'abjurations indiquent pourtant qu'elles avaient déjà abjuré depuis plusieurs années : Marie-Esther Doyerre le 8 juin 1768, et Marie-Luce-Benoîte Gaillard le 20 août 1769 en l'église des Nouvelles Catholiques de Rouen ${ }^{31}$. Marie-Esther Doyerre et son grand-frère, Daniel-David-Paul, ne pouvaient sans doute pas retourner au domicile familial dans la paroisse Saint-Jacques de Dieppe, car leur mère, qui était veuve, avait elle aussi dû abjurer en $1762^{32}$. La petite fut emmenée chez les Nouvelles Catholiques cette année-là, conformément à la législation selon laquelle les enfants d'une veuve protestante devaient lui être retirés pour être élevés dans la religion catholique. Néanmoins, il est difficile d'expliquer pourquoi ni Marie-Esther Doyerre ni Marie-Luce-Benoîte Gaillard ne purent quitter la maison après leur dixhuitième anniversaire, qui correspondait à l'âge moyen de la sortie des pensionnaires ${ }^{33}$. La sœur aînée de cette dernière, Marie-Anne Gaillard, avait quitté la communauté après son abjuration dans l'église des Nouvelles Catholiques, le 23 août 1767, à l'âge de 16 ans ${ }^{34}$. La cadette, Marie-Madeleine, rejoignit Marie-Luce-Benoîte chez les Nouvelles Catholiques en 1772, et s'y trouvait toujours, comme sa sœur, en 1778, bien qu'elle eût abjuré le 18 août 1775, l'année de ses quatorze ans. La durée moyenne du placement semble s'être allongée d'un an au cours du siècle. Elle passa en effet de trois ans et un mois au début du XVIII ${ }^{\mathrm{e}}$ siècle, d'après les états de pensionnaires de 1720 à 1725, à quatre ans et onze mois dans la seconde moitié du siècle, entre 1750

28. Arch. nat., TT 296, dossier 5 .

29. Constitutions pour la maison des Nouvelles Catholiques de Rouen..., p. 30 (chap. II, art. 1).

30. Marie-Catherine Du Seau entra chez les Nouvelles Catholiques de Rouen à l'âge de 12 ans et elle y demeura jusqu'à ses 22 ans, à la fin de l'année 1776. Catherine-Véronique Muloy y demeura neuf ans, de 1768 à 1777, et sa sœur Marie-Marguerite Muloy, d'un an son aînée, y resta plus de dix ans, puisqu'elle figurait encore sur l'état des pensionnaires de 1778 alors qu'elle avait 21 ans.

31. Arch. dép. de la Seine-Maritime, D 487.

32. Ibid. Marie-Élisabeth-Esther et Daniel-David-Paul étaient les enfants de Paul-Pierre Doyerre et Marie-Madeleine Durant.

33. L'âge moyen des pensionnaires au moment de leur sortie était exactement de 18 ans et 11 mois (entre 1720 et 1725) ou 18 ans et 8 mois (pour la période de 1750 à 1778), ce qui montre une réelle stabilité dans la conduite de la maison tout au long du siècle.

34. Arch. dép. de la Seine-Maritime, D 487. L'absence de trace de l'éventuel placement de Marie-Anne Gaillard dans cette communauté s'explique par les lacunes de la série des états de pensionnaires entre 1764 et 1769. 
et 1778 . Ce constat rejoint la question de la mauvaise utilisation des pensions prises sur le Trésor royal, qui fut justement soulevée par le Conseil du roi et aboutit à la rédaction d'un nouveau règlement sur cet usage en 1747. Il était reproché aux supérieures des maisons des Nouvelles Catholiques de garder trop longtemps des jeunes converties au lieu de libérer des places plus fréquemment pour accueillir celles qui étaient encore dans "l'hérésie » ${ }^{35}$. La même année, le secrétaire d'État Louis Phélypeaux, comte de Saint-Florentin, relança les intendants de Normandie pour faire enlever les garçons de 12 à 16 ans et les filles de 9 à 13 ans qui étaient protestants et dont les parents professaient cette même religion ${ }^{36}$. L'âge moyen au moment du placement était en général situé vers 11 ans ${ }^{37}$, mais les ordres du roi ont concerné majoritairement des filles et des jeunes femmes âgées de 5 à 25 ans ${ }^{38}$. $70 \%$ des pensionnaires étaient seulement âgées de 5 à 11 ans au moment de leur arrivée chez les Nouvelles Catholiques. Pourtant, l'âge moyen au moment de leur abjuration s'élevait à 17 ans d'après les registres d'abjurations des Nouvelles Catholiques de 1718 à $1791{ }^{39}$. Au XvirI ${ }^{\mathrm{e}}$ siècle, les plus jeunes abjurantes dans l'église des Nouvelles Catholiques de Rouen furent Marie-Anne-Henriette Prix, petite calviniste de Dieppe âgée de 7 ans, le 9 novembre 1742, et Barthélemie Fortier, Anglaise de confession anglicane, du même âge, le 21 août 1750. La diminution de l'âge d'entrée dans la maison au cours du siècle allait de pair avec l'allongement de la durée du placement, puisqu'il fallait attendre que la nouvelle convertie soit en âge d'être mise en apprentissage ou d'être mariée pour sortir.

Cette longue mise à l'écart des petites et des jeunes filles était synonyme d'une forte rupture familiale. Pendant cette période, les enfants n'avaient plus aucun contact avec leurs parents, sous le prétexte que des conversations avec eux auraient pu les endurcir dans leur obstination à ne pas abjurer :

35. Arch. nat., TT 295, pièces $\mathrm{n}^{\text {os }} 318$ à 322 (" règlement pour les maisons des Nouvelles Catholiques dans lesquelles le roy paye les pensions des filles qui y sont instruites par ses ordres sur les ordonnances qui s'en expédient sur le Trésor royal pendant le temps de leur instruction ", 18 mars 1747). Ce règlement concerne la maison d'Alençon.

36. Arch. dép. de l'Orne, C 618, cité par Nicolas CLOLus, « La politique de répression et de conversion de l'intendant d'Alençon Lallemant de Lévignen envers les protestants (1726-1766) : une politique réussie? ", dans Annales de Bretagne et des pays de l'Ouest, t. 113, n 2 (avr.-juin 2006), p. 88.

37. Arch. nat., TT 296, dossier 5. Cette moyenne a été calculée pour les mineures (c'est-à-dire les filles et jeunes femmes de moins de 27 ans).

38. Ibid. Deux femmes majeures furent également placées sur ordre du roi : Marie-Anne Poignant, veuve de Jean Hébert, le 4 juin 1778, à l'âge de 44 ans, et Marie-Madeleine Deshayes, à l'âge de 55 ans, le 20 septembre 1777. À la fin du Xvis siècle, la communauté des Nouvelles Catholiques de Rouen s'occupa d'enfants encore plus jeunes, tels que Judith Remy, placée à 2 ans ! En 1691, elle apparaît dans la liste des nouvelles catholiques, âgée de 6 ans, étant dans l'institut depuis quatre ans, parce que ses parents avaient émigré. Voir arch. dép. de la SeineMaritime, D 515, mémoire contenant l'état des dépenses et des recettes, un inventaire des biens et un catalogue des personnes de la maison des Nouvelles Catholiques de Rouen, 1691.

39. Arch. dép. de la Seine-Maritime, D 487 et 488. Cette moyenne a été calculée sur les deux cent cinquante-neuf cas d'abjurations de mineures (filles et jeunes femmes de moins de 27 ans). L'âge moyen à l'abjuration s'élevait à 19 ans et demi si l'on prend en compte les trois cents abjurantes recensées dans ces deux registres. 
«Si les hérétiques sont capables d'ébranler la fermeté des filles converties à la foy, par leurs menaces et par leurs mauvais traitements, ils ne sont pas moins à craindre par les recherches et par les conversations douces et amiables qu'ils emploient pour les rapprocher d'eux. On ne peut pas refuser aux filles la liberté de voir leurs parents, mais il est dangereux de les engager trop tôt à avoir des conversations avec eux ${ }^{40}$."

Toute sortie leur était également interdite dans les premiers temps de leur retraite :

"On ne permettra donc point aux filles réfugiées dans la maison de voir leurs parents qu'elles ne soient un peu affermies et fortifiées dans leurs bons desseins; encore ne les verront-elles point qu'elles n'aient avec elles des sœurs capables de les soutenir, et pourtant et si peu de temps que la supérieure verra bon être.

"Elles ne sortiront point de la maison dans le commencement de leur retraite, n'y étant allées que pour s'instruire et pour profiter des exercices qui s'y font, qui leur seraient inutiles si elles avaient la liberté de sortir ${ }^{41}$."

Tout était mis en œuvre pour éviter qu'elles ne revivent avec leurs parents, même après leur abjuration, soit en mettant en métier les jeunes filles chez un maître catholique dans une paroisse différente de celle d'où elles venaient, soit en arrangeant un mariage avec un catholique. Durant cette mise à l'écart, les filles étaient peu à peu persuadées par les sœurs que leurs parents n'éprouvaient à leur égard que de la haine et de la colère. Un tel sentiment d'abandon, réel ou insinué dans leur esprit sans fondement véritable, engendrait bien des conversions résignées. Certes, il est avéré que certaines familles protestantes se détournèrent de leurs enfants qui avaient abjuré. Parfois, les parents avaient émigré, si bien que les enfants se retrouvaient abandonnés et sans ressources ${ }^{42}$. Cependant, les archives comportent aussi quelques lettres de parents accablés par le chagrin qui suppliaient les autorités de récupérer leurs enfants, mais leurs démarches furent sans aucun doute tenues secrètes vis-à-vis des enfants ${ }^{43}$.

C'est donc au terme d'une longue mise à l'écart que les jeunes filles finissaient par se convertir. Placées dans des instituts catholiques, ayant subi une épreuve affective intense — la séparation familiale — , puis le carcan de l'institution religieuse et la pression psychologique exercée par les sœurs ${ }^{44}$, leur conversion pourrait être assimilée à une forme de libre soumission ou de conformisme par intériorisation. Ces concepts issus de la psychologie

40. Constitutions pour la maison des Nouvelles Catholiques de Rouen..., p. 36-37 (chap. II, art. 4).

41. Ibid

42. En 1691, Anne Teuclin (17 ans), Esther d'Angicourt (18 ans), Judith Remy (6 ans) et Marie-Anne de Vaubrun (8 ans) étaient comme abandonnées dans la communauté des Nouvelles Catholiques de Rouen, puisque leurs parents étaient passés à l'étranger (arch. dép. de la Seine-Maritime, D 515, mémoire contenant l'état des dépenses et des recettes, un inventaire des biens et un catalogue des personnes de la maison des Nouvelles Catholiques de Rouen, 1691).

43. A. Joblin, Dieu, le juge et l'enfant..., p. 123-126.

44. Pour la période antérieure à la révocation de l'édit de Nantes, Catherine Martin parle d'un " mélange de persuasion et d'achat des consciences " comme moyens utilisés par les compagnies de la Propagation de la foi pour obtenir des conversions (C. Martin, Les compagnies de la Propagation de la foi..., p. 509). 
sociale ${ }^{45}$ désignent l'attitude des individus qui ont le sentiment de faire un choix délibéré, mais qui ont été en réalité influencés, manipulés au moyen d'armes psychologiques ${ }^{46}$. Dans cette situation, la doctrine soutenue par la majorité est alors intériorisée au point que l'individu, convaincu par ce qu'il a entendu ou vu, n'a pas l'impression de se conformer, mais d'adhérer de son plein gré. L'intérêt de cette hypothèse explicative est de proposer une notion intermédiaire au cœur du débat beaucoup trop binaire entre conversions forcées et conversions volontaires.

Après avoir été arrachées à leur foyer, la conversion de ces filles et de ces femmes renforçait finalement leur rupture familiale. Peut-être faut-il, pour comprendre l'attitude des parents réformés, prendre conscience que le fait de vivre dans la clandestinité n'était pas sans danger, et que le fait d'assumer et de conserver leur appartenance au culte réformé était une fierté familiale ${ }^{47}$. Ce rejet pose donc la question de la définition de l'identité à l'époque moderne. Les nouvelles converties passaient du statut de filles, nièces, épouses, amies à celui d' "étrangères ». Marie-Thérèse Poulain, âgée de 21 ans, femme du sieur Henry Bretot, fille de Jean Poulain et Louise Morel, de la paroisse SaintÉtienne-en-Auge, calviniste, abjura dans l'église des Nouvelles Catholiques de Rouen le 26 septembre 1727 :

«L'abjuration de cette femme avait non seulement indisposé contre elle sa tante, de qui elle était obligée de se cacher pour ses exercices de piété, mais l'a entièrement perdue dans la famille et dans l'esprit de son mari, qui, ayant trouvé le moyen de la dépouiller de son contrat de mariage, crut que les liens qui l'avaient uni avec elle étaient entièrement rompus et, au mépris du sacrement qui les avaient joints, ne la regarde plus que comme une étrangère, rebutant toutes les démarches qu'elle faisait faire pour tâcher de se remettre avec lui, la fuyant de tout côté et l'évitant au point de changer de demeure et de domicile lorsqu'il soupçonnait qu'elle était informée de l'endroit où il habitait ${ }^{48}$."

Quant à sa belle-sœur, elle lui portait une «implacable haine [...] à cause de sa conversion à la religion catholique " ${ }^{49}$. Ces faits conduisent à se demander si l'appartenance religieuse était un élément constitutif de l'identité plus fort que l'appartenance familiale.

45. Trois types de conformisme ont été définis par Herbert C. Kelman en 1958 : le conformisme par complaisance, le conformisme par intériorisation et le conformisme par identification. Voir Dominique Oberlé, "Vivre ensemble : le groupe en psychologie sociale », dans Catherine Halpern (dir.), Identité(s) : l'individu, le groupe, la société, nouv. éd., Auxerre, 2009, p. 135142 .

46. Voir William SARgant, Physiologie de la conversion religieuse et politique, trad. fr., Paris, 1967 (Bibl. de psychiatrie), p. 70-100.

47. Les livres de famille sont les témoins de cette mémoire familiale entretenue et donnée en exemple aux enfants dans les familles huguenotes. Voir D. Solfaroli Camillocai, «Miroirs de vies : identité spirituelle et conscience historique dans la mémoire familiale des réformés entre xvi et xvis siècle ", dans Philip Benedict, H. DAussy et Pierre-Olivier LÉchot (éd.), L'identité huguenote : faire mémoire et écrire l'histoire ( $X V I^{\mathrm{e}}-X X I^{\mathrm{e}}$ siècle), Genève, 2014 (Publications de l'Association suisse pour l'histoire du Refuge huguenot, 9), p. 201-222.

48. Arch. dép. de la Seine-Maritime, D 525, " mémoire pour Marie-Thérèse Poulain, veuve du sieur Henry Bretot, contre la veuve du feu sieur Nicolas Vauloger, sa belle-sœur », s. d. [vers 1750]. Nous soulignons.

49. Ibid. 


\section{La rupture sociale : l'exclusion des nouvelles converties des solidarités confessionnelles}

Une autre forme de marginalisation, induite non pas par un enlèvement, mais par un abandon, pouvait conduire à la conversion au catholicisme. Les six demoiselles Green Mathews se retrouvèrent sans ressources à la mort de leur père, le chevalier Mathews, capitaine des gardes du roi d'Angleterre, qui décéda le 18 septembre 1784 au château des Alleurs ${ }^{50}$. Il avait quitté Londres, certainement en 1771, pour se fixer en France, au nord de Rouen ${ }^{51}$, où il put jouir d'une pension de trente mille livres. La femme du défunt, Elizabeth Maiden ${ }^{52}$, repartit en Angleterre quand elle s'aperçut que la vente des biens du chevalier serait insuffisante à couvrir les dettes qu'il avait contractées par sa "dissipation ». Abandonnées à leur triste sort, les six orphelines attirèrent « la compassion de quelques personnes qui leur donnèrent provisoirement un asile ", puis furent aidées par un ecclésiastique qui, grâce aux aumônes de personnes bienfaisantes, les plaça dans des couvents rouennais en 1784: quatre chez les bénédictines de Saint-Louis (Élisabeth, Charlotte, Pénélope, Cécile), où elles abjurèrent en 1785, et les deux plus jeunes chez les Nouvelles Catholiques ${ }^{53}$ (Marie-Louise, âgée de 14 ans, et la cadette Sophie, qui n'avait que 8 ans en 1784 au moment du placement ${ }^{54}$ ). En 1786, elles demandèrent une pension royale, seule solution envisagée pour survivre dans le monde après leur sortie. Leur requête fut appuyée par certains protecteurs, qui vantèrent leur éducation et leur intégrité :

"Fidèles à l'honneur et à l'honnêteté, elles ont préféré l'indigence à tous les avantages que la corruption s'empressait de leur offrir. Maîtresses d'elles-mêmes, elles se sont rappelées plusieurs fois que deux ecclésiastiques respectables leur avaient dit souvent qu'elles avaient trop de vertu pour n'être pass catholiques un jour : elles ont demandé librement à rentrer dans le sein de la vraie Église ${ }^{55}$. "

50. Ce cas est connu grâce à la correspondance de l'intendant de la généralité de Rouen avec le comte de Vergennes, qui lui avait demandé des renseignements à leur sujet à la suite de la demande de pension royale qu'elles avaient formulée pour leur subsistance (1784-1786). Voir arch. dép. de la Seine-Maritime, C 978, pièces $n^{\text {os }} 171$ à 183.

51. Le chevalier Mathews résida dans la paroisse d'Isneauville (où naquit sa dernière fille, prénommée Sophie, en 1776), dans la paroisse de Bracquetuit et au château des Alleurs. Voir ibid., pièces $\mathrm{n}^{\text {os }} 172$ et 173 .

52. Elizabeth Maiden était la mère de Sophie Green Mathews, née en France, mais non des cinq autres demoiselles Green Mathews, nées près de Londres entre 1764 et 1770.

53. Arch. dép. de la Seine-Maritime, C 978, pièces n ${ }^{\text {os }} 171$ à 173.

54. Arch. dép. de la Seine-Maritime, D 488, et C 978, pièce $\mathrm{n}^{\circ} 177$ (lettre de l'intendant au comte de Vergennes, du 13 décembre 1785, dans laquelle il écrit que la cadette Sophie manifestait "une grande envie d'embrasser la religion catholique", cherchant ainsi à montrer que les renseignements pris justifiaient l'exécution de l'ordre du roi).

55. Ibid., pièce $\mathrm{n}^{\mathrm{o}}$ 171. Ces renseignements paraissent avoir été fournis par $\mathrm{M}^{\mathrm{me}}$ de Canteleu, qui fut peut-être une de leur protectrice. Le document se termine par la phrase suivante : «Leur protection puissante auprès du roi assurera le succès de leur demande. " On peut supposer que c'était le cardinal de La Rochefoucauld qui était ici implicitement désigné, car il fut archevêque de Rouen de 1759 à 1791, résida plus souvent à Paris qu'à Rouen en raison de sa mission au sein de la commission des secours, et l'on sait avec certitude qu'il agit pour les demoiselles Green Mathews d'après la lettre de l'intendant au comte de Vergennes datée du 13 décembre 1785 (ibid., pièce $\mathrm{n}^{\mathrm{o}} 177$ ). 
Marie-Louise et Sophie Green Mathews abjurèrent ensemble le 21 octobre 1787, respectivement âgées de 17 ans et demi et 11 ans et demi ${ }^{56}$. La conversion des jeunes filles avait "indisposé contre elles la famille Mathews », qui «leur refus[ait] tout secours». Ces jeunes filles anglicanes furent donc prises en charge par les structures catholiques d'assistance et soutenues par les fonds royaux, mais cette aide avait été conditionnée par leur conversion. De nombreux convertis, dont le changement de religion provoquait souvent une rupture avec le cercle familial, synonyme de paupérisation, sollicitèrent auprès du roi de France une pension : les preuves sont abondantes dans la correspondance des intendants et les registres de pensions et gratifications royales.

Comment expliquer que les orphelins non catholiques n'aient pas été pris en charge par les œuvres protestantes - qui prenaient souvent la forme de caisses de secours dédiées aux nécessiteux — , ce qui aurait pu éviter leur conversion ? En effet, les réseaux de solidarités confessionnelles avaient pour enjeu de préserver en leur sein les éléments les plus fragiles et d'éviter une conversion au catholicisme induite par leur prise en charge par les catholiques. Les œuvres charitables permettaient notamment de retenir dans la communauté les personnes les plus vulnérables et les plus susceptibles de se tourner vers les structures d'assistance catholiques: veuves, vieillards, enfants, malades, infirmes ${ }^{57}$. Le lien était étroit entre les réseaux protestants de charité et les réseaux de clandestinité religieuse : l'entraide conditionnait «la survie du protestantisme, et non plus simplement des personnes ${ }^{58}$. Cependant, on peut se demander si les divisions internes entre les réformés eurent pour conséquence l'exclusion des anglicans et des luthériens des œuvres charitables des calvinistes ${ }^{59}$. Ces formes d'entraide confessionnelle, dont le sens même reposait sur la perpétuation de leur propre communauté religieuse, semblaient avoir un caractère exclusif. C'est peut-être là que réside la clé de compréhension de la marginalisation de certains enfants — telles les demoiselles Green Mathews, qui étaient anglicanes —, et a fortiori celle de l'exclusion des nouveaux convertis des solidarités confessionnelles après leur

56. Arch. dép. de la Seine-Maritime, D 488.

57. Natalia Muchnik, "Charité et communauté diasporique dans l'Europe des xvi xvirI ${ }^{\mathrm{e}}$ siècles", dans Revue d'histoire moderne et contemporaine, t. 6l, $\mathrm{n}^{\mathrm{o}} 3$ (juil.-sept. 2014), p. 7-27. Natalia Muchnik mentionne la création, à Amsterdam, le 12 février 1615, de la Santa Companhia de dotar orfans e donzelas pobres, confrérie de la congrégation judéo-ibérique chargée de fournir une dot aux orphelines et jeunes filles pauvres de la diaspora séfarade " résidant de Saint-Jean-de-Luz à Dantzig ». La nation des juifs portugais s'organisait autour de la Sedeca, société de bienfaisance fondée sur un système de cotisations obligatoires, qui distribuait des secours (soins et nourriture) aux pauvres malades, leur octroyait la gratuité du bain rituel et finançait des écoles juives. Cependant, il n'existait pas toujours d'œuvres particulières pour les enfants juifs abandonnés. À Bordeaux, ils étaient placés dans les hôpitaux catholiques, tel l'hôpital Saint-Louis (Jean Cavignag, Les israélites bordelais de 1780 à 1850 : autour de l'émancipation, Paris, 1991 (La France au fil des siècles), p. 313).

58. Céline Borello, «Introduction. Secourir dans la tradition protestante du xvi au $\mathrm{XIX}^{\mathrm{e}}$ siècle : les œuvres comme exigence liturgique, ordre politique et lien social ", dans EAD. (éd.), Les œuvres protestantes en Europe, Rennes, 2013 (Histoire), p. 9-22.

59. Cette question est suggérée par le cas des sociétés de bienfaisance des juifs portugais qui ne venaient pas en aide aux juifs tudesques, avignonnais, italiens ou levantins (N. MuGHNIK, "Charité et communauté diasporique...", p. 11). 
abjuration, quand bien même celle-ci était obtenue de force. En somme, aux $\mathrm{xVII}^{\mathrm{e}}$ et $\mathrm{xVIII}{ }^{\mathrm{e}}$ siècles, l'extraction des jeunes filles de leur cercle de socialisation primaire par des enlèvements ou des placements dans des couvents catholiques, et l'absence de prise en charge des orphelines par les réseaux de charité de leur groupe confessionnel d'origine constituaient une forme de marginalisation sociale aboutissant à leur conversion au catholicisme. Celle-ci ne faisait qu'accentuer le rejet de la part de leur famille et, à une autre échelle, de la part de la minorité religieuse. La mobilisation des structures d'assistance sociale - hôpitaux surtout, mais aussi couvents acceptant de recevoir des pensionnaires - peut être également envisagée comme une méthode servant les intérêts de la religion : venir au secours des non-catholiques, quel que soit leur sexe ou leur âge, afin d'apparaître comme leur seule voie de salut... Cependant, les itinéraires de nouvelles converties mettent en évidence une spécificité féminine dans les stratégies utilisées par les catholiques pour convertir les jeunes filles : la construction de leur isolement pour les amener à renier leur foi première.

Dans le cas des demoiselles Green Mathews, si leur conversion paraît volontaire, elle pourrait être aussi qualifiée de «conversion instrumentalisée ", « stratégique " ${ }^{60}$ ou " négociée » ${ }^{61}$. En effet, le besoin de secours matériels a motivé de nombreuses conversions au catholicisme dans des contextes très divers ${ }^{62}$. La conversion pouvait aussi revêtir un caractère juridique, car le statut de catholique conférait des droits civils en matière de jouissance de la propriété et de transmission des biens, outre la sécurité qu'il donnait aux personnes. Sous le règne de Louis XIV, la majorité des abjurations furent forcées ou bien résignées, étant donné le rapport de force religieux, politique et militaire en faveur des catholiques. Le plus grand nombre d'abjurations coïncide justement avec les pics de persécutions, pendant les dragonnades de 1681 à 1683 puis après la révocation de l'édit de Nantes en 1685 et 1686. Dans un climat de terreur ambiant, la peur de la répression a conduit les membres de ces minorités en danger, subissant des vexations, des injustices, à se convertir au catholicisme. Pourtant, il y a eu aussi, en France, de 1685 à 1787, un certain nombre de conversions volontaires au catholicisme. L'existence de conversions relevant d'une véritable conviction spirituelle, d'un changement de religion sincère et assumé, est indéniable. Elle remet d'ailleurs en cause

60. La notion de « conversion stratégique » est employée par Jérémie Foa au sujet des hommes du culte réformé ayant abjuré dans le but de conserver leurs biens à la fin du Xvi ${ }^{\mathrm{e}}$ siècle (Jérémie FoA, "Dans les petits papiers du pouvoir : abjurations huguenotes du temps de l'édit de Nemours (1585) ", dans T. Lienhard et I. Poutrin (éd.), Pouvoir politique et conversion religieuse..., t. I, en ligne à l'adresse : http://books.openedition.org/efr/3476 [site consulté le 3 mars 2018]).

61. C. Martin, Les compagnies de la Propagation de la foi..., p. 158-159. Outre leurs controverses pour convertir les hérétiques et leur rôle de surveillance, les compagnies de la Propagation de la foi offraient une aide matérielle aux nouveaux convertis : « La conversion faisait l'objet d'une négociation préalable. Les avantages matériels et sociaux devaient y prendre une place importante " (ibid., p. 159).

62. Voir Thomas GLesENER, «Se (re)faire musulman : l'accès des pauvres itinérants aux droits de la conversion (Espagne, xviI ${ }^{\mathrm{e}}$ siècle) ", dans Revue d'histoire moderne et contemporaine, t. 64, nº 2 (avr.-juin 2017), p. 129-156, et Monica MARTinAt, "Genevois à Lyon, Lyonnais à Genève : itinéraires de migrants et de convertis (xvi' ${ }^{\mathrm{e}}$ siècle) ", dans Revue de l'histoire des religions, t. 232 (2015), p. 37-51. 
l'idée d'une appartenance religieuse statique et nuance le postulat de la perpétuation d'une identité religieuse immuable au sein d'une famille. D’autres conversions apparemment volontaires semblent avoir été motivées par le désir de s'intégrer dans une société française qui était alors exclusivement catholique. On peut tenter de comprendre cette posture, consistant à conserver ou nouer des relations positives avec la majorité catholique, en invoquant la notion de «conformisme par identification " ${ }^{63}$. Cette catégorie pourrait s'appliquer aux personnes ayant accepté de se convertir au catholicisme parce qu'elles cherchaient à s'insérer dans un réseau de sociabilité catholique, en vue d'accéder à un office royal ou pour nouer une alliance matrimoniale avec une famille catholique ${ }^{64}$. Le changement de religion devenait par conséquent un outil d'intégration sociale. La conversion au catholicisme, aux XVII et XVIII ${ }^{\mathrm{e}}$ siècles, peut donc se comprendre comme un ensemble de désirs complexes, plus ou moins inspirés par un élan spirituel et religieux, mais souvent aussi attisés par des intérêts pragmatiques variés : motivations sociales (conclure une alliance, accéder à un office réservé aux catholiques), juridiques (garantir la sécurité de sa personne et de sa famille, de son statut et de ses droits), économiques (commercer librement, transmettre son entreprise à sa famille), ou bien encore des arguments politiques ${ }^{65}$. Certes, figer une typologie des conversions, ce serait omettre que tout un faisceau de causes entrait en jeu pour chacune. L'historien ne peut avoir aucune certitude lorsqu'il travaille sur l'intentionnalité des acteurs, en raison de la complexité de chaque personnalité, quand bien même les sources seraient exhaustives à son sujet. Néanmoins, le croisement des concepts entre les sciences humaines permet de proposer de nouvelles hypothèses pour analyser, dans les sociétés passées, les comportements individuels en les réinscrivant dans des logiques de groupe. Finalement, une partie des nouveaux convertis de Normandie auraient donc accepté de se convertir dans le but de s'intégrer au groupe le plus fort, le plus nombreux, quitte à prendre le risque d'une rupture familiale et d'un rejet de la part du groupe confessionnel d'origine. La raison de ces types de conversion paraît être plus forte que la simple "pratique d'une sociabilité quotidienne » ${ }^{66}$; il s'agirait d'une réponse à une véritable nécessité de s'intégrer.

63. L'individu accepte de se conformer parce qu'il s'identifie à un groupe et qu'il veut être accepté en son sein.

64. C. Martin, Les compagnies de la Propagation de la foi..., p. 159 et 378-395. Les compagnies de la Propagation de la foi se donnaient pour mission d'intégrer les nouveaux catholiques dans leur nouveau réseau de solidarité en faisant jouer leurs contacts pour les aider à conclure un mariage, pour trouver un emploi ou pour obtenir des lettres de maîtrise.

65. É. SurRe, "L'argument politique dans les récits de conversion français du xviI siècle ", dans P. Martin et É. Suire (éd.), Les convertis..., t. I, p. 175-189.

66. L'expression est proposée par Élisabeth LABrousse, "Conversion dans les deux sens", dans La conversion au XVII siècle : actes du XII colloque de Marseille (Centre méridional de rencontres sur le XVII siècle, janvier 1982), [Marseille], 1983, p. 161-177. Voir également Estelle Martinazzo, «Se convertir ou fuir : la question protestante dans le diocèse de Toulouse (15801685) ", dans Cahiers d'études du religieux, ${ }^{\circ}$ spécial (2011) ["Actes de la journée Jeunes chercheurs sur la conversion "], en ligne à l'adresse : http:// cerri.revues.org/768 [site consulté le 3 mars 2018]. 


\section{Une réinsertion sociale médiatisée par la reconstruction d'une appartenance religieuse}

De 1685 à 1787, entre la révocation de l'édit de Nantes et l'édit de tolérance ${ }^{67}$, les nouveaux catholiques ont été maintenus, aux yeux de l'État et de l'opinion publique, dans un statut ambigu : sujets dotés de droits civiques et juridiques, intégrés économiquement et socialement, ils étaient néanmoins suspectés de dissimuler leur foi véritable et d'être restés intérieurement fidèles au culte réformé malgré leur abjuration ou celle de leurs parents ou grands-parents. Par exemple, Anne-Marguerite Carnou, originaire d'Elbeuf, fut placée le 27 juin 1751 chez les Nouvelles Catholiques de Rouen ${ }^{68}$ et ne sortit qu'au bout de dix ans, à la fin de l'année 1761, à l'âge de 19 ans, alors qu'elle avait abjuré depuis sept ans, le 18 août 1754 d'après le registre des abjurations ${ }^{69}$. Comment expliquer cette privation de liberté après le temps de son instruction, après son abjuration et sa communion, comme tant d'autres? Les critères de sortie des maisons de Nouvelles Catholiques étaient mal définis dans les Constitutions, ce qui pouvait entraîner des périodes très longues de maintien dans l'institut. Après les trois mois — théoriques — d'instruction, les nouvelles converties devaient être remises « entre les mains des personnes qui les [avaient] présentées» ${ }^{70}$ ou à "quelque personne de probité connue " ${ }^{71}$. Mais comment cette condition pouvait-elle être remplie lorsque les enfants avaient été enlevés à leurs parents ? Sous l'apparence d'un refuge, d'un asile, la communauté pouvait trouver des arguments pour empêcher ces enfants de rejoindre un milieu resté calviniste :

«L'on y pourra [...] garder quelquefois quelques-unes de celles qui, étant instruites, sont persécutées et affligées par leurs parents hérétiques, et abandonnées de tout secours, et on les gardera jusqu'à ce qu'on leur ait procuré quelque asile et quelque retraite sûre, ce qui ne se fera pourtant qu'avec beaucoup de prudence et de discrétion, pour ne point charger la maison, et ne pas occuper la place de celles qui ont besoin d'instruction ${ }^{72}$."

L'anthropologie apporte un éclairage sur le statut ambigu des filles ou jeunes femmes qui demeuraient dans l'institut alors que le temps de leur instruction était terminé depuis longtemps. Cette période d'attente avant leur

67. L'édit de Versailles de 1787 accorda un état civil aux non-catholiques en légalisant les mariages non religieux conclus en présence d'un juge royal ou d'un curé de la paroisse agissant comme officier civil. Les naissances et les décès pouvaient être enregistrés de la même manière par simple déclaration. Cet édit reconnaissait aux non-catholiques le droit d'exister dans le royaume sans y être troublés sous prétexte de religion, bien que l'accès aux charges publiques et à l'enseignement leur demeurât fermé et que la prédominance de la religion catholique fût réaffirmée.

68. Arch. nat., TT 296, dossier 5, pièce $\mathrm{n}^{\circ} 110$ (état des Nouvelles Catholiques de Rouen pour le «troisième quartier » de l'année 1751).

69. Arch. dép. de la Seine-Maritime, D 487.

70. Constitutions pour la maison des Nouvelles Catholiques de Rouen..., p. 39-40 (chap. II, art. 6).

71. Ibid., p. 28-30 (chap. II, art. 1).

72. Ibid. 
retour dans le monde, par la mise en apprentissage de l'adolescente ou le mariage de la jeune femme, peut s' apparenter à une phase de liminarité ${ }^{73}$. La supérieure de la maison des Nouvelles Catholiques devait s'assurer que la conversion de ces jeunes filles était sincère et ferme avant de leur permettre de regagner le monde. Elles étaient donc maintenues dans une sorte de statut intermédiaire entre leur passé d' "hérétiques" et un statut de "nouvelles converties » jugé avéré. Par conséquent, leur intégration sociale dépendait de leur capacité à donner des preuves d'une « véritable » conversion. La mise en apprentissage chez un maître catholique, à partir de 14 ans environ, ou un mariage avec un catholique constituaient une voie de réinsertion dans la société. C'est ainsi qu'en 1691 Anne Teuclin, 17 ans, apprenait le métier de lingère. Marie et Marguerite Robin, respectivement âgées de 13 et 10 ans, faisaient de la dentelle ${ }^{74}$. Les règlements insistaient sur l'obligation de ne fréquenter que d'anciens catholiques :

«Aucune fille nouvelle convertie ne sortira de la maison des Nouvelles Catholiques que pour être mariée à un ancien catholique qui aura un bon métier, auquel cas le roy payera pour sa dotte une somme de 100 livres, ou pour être placée domestique dans une maison d'anciens catholiques ou pour entrer chez une maitresse ancienne catholique du choix de l'évesque diocézain pour y apprendre le métier pour lequel on luy connaîtra le plus de disposition, en observant néanmoins de n'en faire apprendre aux filles de la campagne que de ceux qui y conviennent. La maîtresse donnera sa soumission par écrit de l'envoyer pendant le tems de son apprentissage aux instructions de la maison des Nouvelles Catholiques les jours à ce destinés, d'avoir soin qu'elle assiste au service divin et qu'elle remplisse exactement les devoirs de la religion et, en cas de dérangement, de relations ouvertes ou cachées avec des nouveaux convertis, d'en avertir la supérieure des Nouvelles Catholiques, et celle-cy en informera l'évesque diocézain. [...].

"Après que les filles nouvelles converties auront fait le tems de leur apprentissage, elles seront envoyées dans telles paroisses de la campagne que l'évesque diocézain trouvera le plus à propos, autres que leur propre paroisse pour exercer leur métier ${ }^{75}$. »

Dans ce contexte, la conversion au catholicisme était synonyme d'une redéfinition de l'identité personnelle — et non pas seulement religieuse par la recomposition des liens sociaux qu'elle provoquait. Le changement $\mathrm{d}^{\prime}$ " appartenance religieuse » ${ }^{76}$, qui se traduisait autant par des pratiques que par l'adhésion à une doctrine, prenait la forme d'une affiliation à un nouveau

73. En anthropologie, le concept de liminarité permet de qualifier la phase intermédiaire au cours d'un changement de statut, en particulier lors des rites de passage. La phase de liminarité se situe entre un premier moment de séparation et une dernière étape de réincorporation. Cette notion présente l'intérêt, dans le cas des conversions religieuses, de mettre en relief la rupture sociale induite par celles-ci, et la nécessité de préparer ensuite, durant cette période, l’insertion dans un nouveau réseau social.

74. Arch. dép. de la Seine-Maritime, D 515, mémoire contenant l'état des dépenses et des recettes, un inventaire des biens et un catalogue des personnes de la maison des Nouvelles Catholiques de Rouen, 1691.

75. Arch. nat., TT 295, pièces $\mathbf{n}^{\text {os }} 318$ à 322 (articles 7 et 9).

76. On préférera le terme d'" appartenance religieuse» à celui d' "identité religieuse ». En effet, la notion d' "identité religieuse » est porteuse d'une contradiction, car elle désigne à la fois les éléments qui individualisent une personne et ceux qui sont constitutifs d'une collectivité donnée. Voir Olivier Chaline, "Introduction », dans N. Champ et É. Suire (éd.), Les appartenances religieuses..., p. 11-18. 
groupe religieux, donc à un nouveau groupe social, exclusivement catholique, dans un nouveau village. Mais, après leur sortie, dans quelle mesure les nouvelles converties étaient-elles intégrées en tant que personnes dans la société française? Ne portaient-elles pas toute leur vie le poids de leur identité de transfuges, à moins de faire preuve d'une piété exemplaire ? La suspicion inhérente au statut des nouveaux convertis, jusqu'à l'édit de tolérance de 1787, rendait primordiale l'officialisation du changement de religion de manière ostensible pour légitimer leur nouvelle appartenance sociale et religieuse. À leur sortie du couvent, les nouveaux convertis s'engageaient à se rendre à la messe tous les dimanches, à participer aux fêtes catholiques et à continuer à assister aux instructions hebdomadaires. Ils étaient étroitement surveillés par les curés de paroisse ${ }^{77}$, et les intendants demandaient parfois de prendre des renseignements à leur sujet, car ils étaient toujours suspectés de revenir, un jour, à leur religion première.

Quand certaines retrouvaient le monde, d'autres faisaient le choix ou n'avaient d'autre choix que - de rester entre les murs où elles avaient été placées et élevées, dans une communauté qui les privait donc de liberté, et qui les coupait de la société. Les maisons de Nouvelles Catholiques, et plus généralement les communautés religieuses ${ }^{78}$, pouvaient apparaître comme des refuges pour les filles et les femmes en situation de vulnérabilité. La décision de quelques nouvelles converties de demeurer dans un couvent catholique peut s'expliquer par une longue mise au ban de la société, un sentiment d'abandon, la crainte d'être rejetée, la peur du retour dans la société auquel elles n'auraient pas été préparées, le manque de ressources, l'absence de moyen de subsistance, qui pouvait les pousser à demander une pension au roi pour demeurer dans la communauté. L'enfermement dont étaient victimes des jeunes filles protestantes, l'isolement orchestré pour les conduire inexorablement à leur conversion pouvaient aboutir à des vocations forcées ou résignées ${ }^{79}$, l'entrée en religion restant la seule perspective pour quelques jeunes filles privées de contact avec leur famille ou n'ayant plus d'attache familiale. Ces nouvelles converties devenues religieuses dans des couvents catholiques qualifiaient parfois elles-mêmes leur

77. En revanche, dans la capitale du royaume, il était beaucoup plus aisé d'échapper à la surveillance des curés dans la seconde moitié du xviII ${ }^{\mathrm{e}}$ siècle. Peu à peu, la paroisse joua un rôle de moins en moins actif dans la vie des Parisiens, que ce soit en tant qu'unité d'organisation sociale, socle identitaire, instance moralisatrice, dont le rôle de régulation était laissé à la police. Voir David Garrioch, La fabrique du Paris révolutionnaire, trad. fr., Paris, 2013, p. 206-207.

78. Les sources d'autres couvents rouennais corrèlent et complètent celles de la maison des Nouvelles Catholiques de la ville. Sur le rôle de refuge des communautés religieuses féminines à Rouen, voir A. LoRIAud, Les clefs du cloître : les communautés de femmes à Bordeaux et Rouen aux $X V I I^{e}$ et $X V I I I^{e}$ siècles, thèse de doctorat en préparation sous la direction de M. Éric Suire, à l'université Bordeaux-Montaigne.

79. Les entrées en religion des filles catholiques pouvaient aussi apparaître parfois comme étant « conditionnées » (par les religieuses du pensionnat dans lesquelles elles avaient grandi, ou par d'autres ecclésiastiques, ou par un membre de la famille entré dans un couvent), ou bien résignées, comme une soumission à l'autorité parentale, ou un choix par défaut dans une situation complexe (perte d'un parent, échec d'un mariage). L'époque moderne n'autorisait pas réellement de libres choix de vie, y compris pour les mariages ou la carrière professionnelle. Voir D. DinET, Vocation et fidélité..., p. 15-47 et 276-277. 
conversion de « sincère » ${ }^{80}$, ce qui n' est pas impossible bien sûr, mais il est difficile de ne pas voir dans leur choix une part de crainte, d'abord vis-à-vis de leurs parents, ensuite par rapport au monde extérieur, craintes sans doute renforcées par l'emprise que les religieuses avaient sur leur esprit. Le rôle de refuge de l'institut des Nouvelles Catholiques est confirmé par les demandes émanant d'adolescentes et de jeunes femmes protestantes sollicitant d'elles-mêmes des ordres du roi pour entrer dans une communauté religieuse. La détermination de Suzanne Prévost est à cet égard éloquente. La jeune fille, originaire du Havre, écrivit une lettre, le 17 mars 1785, à la supérieure des Nouvelles Catholiques de Rouen avant de s'enfuir secrètement de chez ses parents :

«Madame, je suis restée tranquille ici jusqu'à présent parce que mes parents se proposaient de me mettre en pension à Rouen et que là j'aurais été à portée d'entrer plus facilement chez vous (ce que monsieur le curé du Havre avait approuvé), mais il paraît aujourd'hui que ce voyage n'aura pas lieu, ce qui me forcera d'employer ici les moyens que vous voudrez bien m'indiquer pour y parvenir. Je vous serai obligée aussi de me faire savoir si vous êtes toujours munie de la lettre du roy qui vous autorise à me recevoir, si, vu le laps du temps qui s'est écoulé, elle est encore valide, et enfin si à quinze ans, que j'aurai le vingt du mois d'avril prochain, je suis la maîtresse d'agir à cet égard, sans crainte de retomber entre les mains de mes parents ${ }^{81}$."

Mais les pensées profondes des auteurs de ce type de témoignage sont insaisissables. Cette jeune fille ne cherchait-elle pas surtout à s'émanciper de la tutelle parentale? Quelques filles et femmes évoquaient, dans leurs lettres ou leurs suppliques, les " mauvais traitements » de la part de leurs parents ou mari protestants pour demander un ordre du roi afin de se réfugier dans un couvent. Pourtant, la nature de l'institut fut un objet de débat au milieu du XVIII ${ }^{\mathrm{e}}$ siècle. Le roi souhaitant mettre un terme aux demandes abusives de pensions payées sur le Trésor royal, il rappela en 1747 aux communautés de Nouvelles Catholiques que les filles placées sur ses ordres pour être converties devaient sortir de la maison trois mois après leur première communion afin de porter les fruits de leur conversion à l'extérieur et de laisser la place à d'autres ${ }^{82}$. Certaines supérieures, soutenues par l'évêque, se défendirent alors en prétextant être dans l'impossibilité de faire sortir des fillettes qui n'avaient pas encore atteint l'âge d'être mises en métier ou dont les parents se trouvaient à l'étranger, ainsi que des jeunes femmes miséreuses ou des infirmes ${ }^{83}$. C'est pourquoi Marie Mulady put demeurer treize ans chez les Nouvelles Catholiques de Rouen, entre 18 et 31 ans, bénéficiant d'une pension du roi de 1745 jusqu'à sa sortie entre septembre 1759 et mars $1760{ }^{84}$. Dans une

80. Arch. nat., TT 264, dossier 16, placet adressé par Marie Le Boulanger et Marie Bunel au marquis de Châteauneuf, 24 mars 1688.

81. Arch. dép. de la Seine-Maritime, C 978, pièce $n^{\circ} 185$ (lettre de Suzanne Prévost à la supérieure des Nouvelles Catholiques, 17 mars 1785).

82. Arch. nat., TT 295, pièces $\mathrm{n}^{\text {os }} 318$ à 322 .

83. Ibid., pièces $\mathrm{n}^{\text {os }} 402$ à 407 (réflexions présentées au ministre par l'évêque de Bayeux sur l'état comprenant les filles et les garçons qui doivent être renvoyés des maisons de Nouvelles et Nouveaux Catholiques de Caen, s. d. [vers 1747-1750]).

84. Arch. nat., TT 296, dossier 5, pièces $\mathrm{n}^{\text {os }} 97$ à 140 (états des Nouvelles Catholiques de Rouen, 1750-1756 et 1759). 
extrême misère depuis la mort de son père, officier irlandais catholique du régiment de Barwick tué à la bataille de Fontenoy en 1745, cette orpheline avait des chances de s'établir convenablement, car « Milord Clarck et bien des personnes qui [avaient] connu le père s'y intéress[ai]ent " ${ }^{85}$. Ce refuge pouvait être choisi dans l'attente d'un avenir meilleur ou pour y passer sa vie entière. Chez les Nouvelles Catholiques, de nombreuses converties prirent à leur tour le rôle d'enseignantes pour instruire dans la foi catholique les petites protestantes. Il était d'ailleurs recommandé, dans les Constitutions, de donner cette charge à des nouvelles converties, partant de l'idée que celles-ci seraient plus aptes à faire face aux contre-arguments des jeunes filles protestantes et pourraient ainsi mieux les convaincre. Pour vérifier la propension des converties à devenir à leur tour des agents de conversion, l'absence de registres de réception des sœurs ${ }^{86}$ peut être partiellement comblée par un registre des délibérations de la fin du XVII ${ }^{\mathrm{e}}$ siècle ${ }^{87}$, et par les signatures apposées par les supérieures sur les registres et divers papiers, notamment les états envoyés pour recevoir les pensions du roi. Par exemple, Suzanne Robin, née dans une famille calviniste et placée dans la maison des Nouvelles Catholiques de Rouen vers 1686, apprit le métier de couturière ${ }^{88}$, mais se tourna vers la vie religieuse, puisqu'elle faisait partie des sœurs de la communauté en $1698{ }^{89}$. Elle en devint même supérieure en $1753{ }^{90}$. En 1792, lorsque les officiers révolutionnaires dressèrent des listes de religieuses, Marie-Anne-Henriette Prix et Marie-Anne-Madeleine Le Tourneur furent recensées parmi les onze sœurs de la communauté des Nouvelles Catholiques de Rouen ${ }^{91}$. Elles étaient toutes les deux nées dans des familles calvinistes : l'une venait de la paroisse Saint-Jacques de Dieppe, l'autre de la paroisse Saint-Herbland de Rouen. Mais si Marie-Anne-Henriette Prix avait été élevée par les sœurs de

85. Arch. dép. de la Seine-Maritime, D 516, avis donnés par l'archevêque de Rouen sur la continuation des pensions des Nouvelles Catholiques de Rouen, 1746.

86. D'après ses Statuts et constitutions, de 1683, la communauté des Nouvelles Catholiques était un institut séculier dans lequel les sœurs, qui conservaient leur statut de laïques, vivaient en commun sans prononcer de vœux de religion (arch. dép. de la Seine-Maritime, D 478, fol. lr). Les sources traditionnelles d'étude des entrées en religion (examens de vocation dans les archives de l'archevêché, contrats d'aumônes dotales dans les archives régulières ou notariales) n'existent donc pas pour cet établissement. Le fonds de la maison des Nouvelles Catholiques de Paris n'est d'aucun apport sur la fondation rouennaise, puisqu'il ne s'agit pas d'une congrégation à supérieure générale dont la maison-mère aurait été à Paris. La maison rouennaise fonctionnait de manière autonome.

87. Arch. dép. de la Seine-Maritime, D 489. Dans les premiers temps de la communauté, du moins entre 1675 et 1698, les réceptions des sœurs étaient inscrites dans le registre des délibérations.

88. Arch. dép. de la Seine-Maritime, D 515, mémoire contenant l'état des dépenses et des recettes, un inventaire des biens et un catalogue des personnes de la maison des Nouvelles Catholiques de Rouen, 1691. Suzanne Robin y est mentionnée ainsi que ses deux petites sœurs (citées plus haut) avec une indication précisant que leurs parents étaient encore protestants.

89. Arch. dép. de la Seine-Maritime, D 489, fol. 50-51. Suzanne Robin faisait partie des cinq sœurs qui signèrent l'acte de réception de Françoise Agasse le 5 septembre 1698.

90. Elle ne semble pas avoir accompli longtemps cette charge, puisqu'elle fut remplacée avant la fin de l'année 1753 par Marie Faulcon. Elle venait peut-être de décéder, car elle avait alors 79 ans. Voir Arch. nat., TT 296, dossier 5, pièces n ${ }^{\text {os }} 119$ à 126 (états des Nouvelles Catholiques de Rouen, 1753-1754).

91. Arch. nat., D XIX 8, dossier 123 (état des religieuses du diocèse de Rouen en 1790), liste des filles séculières de la communauté des Nouvelles Catholiques de Rouen. 
cette communauté dès son plus jeune âge, Marie-Anne-Madeleine Le Tourneur semble y être entrée à l'âge adulte. En effet, Marie-Anne-Henriette abjura le 9 novembre 1742 à l'âge de 7 ans, tandis que Marie-Anne-Madeleine avait déjà 18 ans lorsqu'elle abjura le 18 août 1754 dans l'église des Nouvelles Catholiques ${ }^{92}$. Afin d'expliquer le choix de certaines converties de rester dans la communauté où elles avaient été placées et élevées, il conviendrait de relier ce problème à la question de leur intégration. Cette hypothèse reposerait sur l'idée que leur vocation religieuse arrivait au terme du processus de marginalisation familiale et sociale qu'elles avaient subi et s'expliquerait par leur souhait de reconstruire une appartenance religieuse et sociale, autrement dit leur appartenance à un groupe : celui que constituent les sœurs d'une communauté religieuse. En effet, le couvent était un espace où elles étaient protégées, où elles avaient une place et un rôle au sein de la communauté. Partageant petit à petit les mêmes croyances et les mêmes pratiques, les nouvelles converties adoptaient, en choisissant de rester dans un couvent, une conscience de groupe, poursuivaient des buts communs avec les autres sœurs - la quête de leur salut ou la mission d'enseignement — et étaient intégrées par des interactions avec elles.

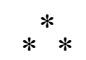

Le cas rouennais, à travers les papiers des Nouvelles Catholiques, met en lumière la complexité des facteurs ayant pu intervenir dans les phénomènes de conversions des jeunes protestantes entre la révocation de 1685 et l'édit de tolérance de 1787. En recontextualisant ces démarches individuelles dans des logiques de groupe, il apparaît qu'une part de ces changements d'appartenance religieuse résultait d'un processus de marginalisation familiale et sociale doublée d'un isolement sciemment construit par ceux qui voulaient les convertir. Aussi longtemps que le processus de conversion n'aboutissait pas à une réinsertion sociale, les nouvelles converties étaient maintenues dans une sorte de liminarité. Accepter son incorporation définitive dans une communauté de sœurs ou de religieuses offrait une perspective de sortie de ce statut ambigu. En définitive, l'entrée en religion dans un couvent, pour une nouvelle convertie, répondrait au désir de se sentir intégrée à un groupe, quitte à rejoindre une autre forme de marginalité sociale, caractéristique des sœurs et des religieuses.

Aude Loriaud,

université Bordeaux-Montaigne,

Centre d'études des mondes moderne et contemporain.

92. Arch. dép. de la Seine-Maritime, D 487. 